\title{
Research on Direct Detection of Dark Matter Based on the Little Higgs Model
}

Feng Zhang

Dalian Institute of Science and Technology, Lvshun, 116052, China

\begin{abstract}
Dark matter is one of the hot spots in the astronomy, but the stand model of the particle physics can't provide a proper candidate of dark matter. There is also a contradiction between the initial little Higgs model and the accurate measurement of weak current. Therefore, the scientists introduced T-parity into the little Higgs model. The paper directly detected the dark matter based on the little Higgs Tparity model to provide some references for the relative researchers.
\end{abstract}

Keywords:little Higgs model, dark matter, direct detection

\section{Concept of Dark Matter}

One of the basic tasks of particle physics is to explore the microscopic origin of the macroscopic material world. The twentieth century, whose representative is the particle physics standard model, is the golden age of particle physics. In the framework of symmetry, the standard model successfully unifies the micro world three basic interactions except gravity, electromagnetic interaction. In other words, from the standard model, almost all of the current laboratory micro physical phenomena can be explained. But in our universe, in addition to the luminous baryonic matter, there are still a lot of bad luminescent substances, namely dark matter. The invisible ghost, filling the universe, subverts the possibility that the standard model is the ultimate theory. To further confirm the existence of dark matter, and then to study the basic properties of dark matter, has become a need for particle physicists and astronomers to shoulder the responsibility of the era of scientific research. It was not until 60 and twentieth Century 70 s that astronomers found that most of the spiral galaxies were faster than the theoretical ones. One of the simplest reasons is that these galaxies contain many invisible masses. Now, scientists on the dark matter definition basically formed a consensus. It is only in gravity, which is not involved in 
electromagnetic interaction, is not currently known to us of any kind of particles or whether it is no conclusive particle. It is generally believed that the main component of dark matter is cold dark matter, that is, the mass of weakly interacting particles. The residual WIMP is consistent with the proportion of dark matter abundance in the universe and in accordance with thecurrent understanding of dark matter particles. It is the most promising candidate to study and verify.

\section{Detection methods of dark matter}

\subsection{Direct detection}

If the dark matter in the galaxy is composed of weakly interacting particles, there are thousands of WIMPs particles per second per square centimeteracross the earth. The detection of dark matter particles depends on the type and intensity of interaction with the standard model particles. WIMP dark matter can be detected by collision with conventional materials. The general assumption the quality of WIMP $\mathrm{m} \mathrm{x}$ is about $100 \mathrm{Ge} \mathrm{V}$ and the speed of $\mathrm{V}$ is about $10^{-3} \mathrm{~m} / \mathrm{s}$. The energy after the collision is about $100 \mathrm{keV}$, so the underground test ultra-sensitive detector. This detector cannot detect the strong interaction of dark matter, dark matter can be because of the atmosphere or rock block, leading to more complex detection more uncertain, comprehensive factors, experiment has ruled out a large cross section. The direct detection of dark matter is very active, which has a rapid development in the past decade detector sensitivity has been improved by two orders of magnitude. The scattering cross section reached $10^{-45}-10^{-44} \mathrm{~cm}^{2}$. Collision of WIMP and nuclear can spin dependent coupling, DAMA coupling or experimental group spin dependent through the observation that perennial signal is cyclical. Some theorists doubt the result of DAMA/LIBRA. If these signals from the spin independent dark matter collisions, so the quality and the scattering cross section of dark matter not in the exclusion of particles below the line. The experimental results measure the scattering cross section of other groups has been eliminated. The results of DAMA are interpreted as inelastic scattering. Spin dependent scattering provides an independent approach to the detection of dark matter. At present, the experimental group in this area has CDMS, COUPP and KIMS, but they are powerless in the limitations of the super symmetric parameter space.

\subsection{Indirect detection}

After dark matter decoupling, the annihilation speed is also greatly reduced. The influence on residual density may be negligibleand the residual density basically unchanged, even so, the annihilation of dark matter does not stop, it is possible to be of indirect detection: somewhere in annihilation. Some other substances can be detected directly. Many methods have been devised to rely heavily on the components of the WIMP, the system error, and the background. A typical method is to detect neutrinos produced by the annihilation of dark matter. When 
the WIMPs through the sun or earth between them collide with each other, while the speed is reduced to the escape velocity, which is the sun or the earth's capture, they may stay to accumulate in the interior of the earth, and the density of WIMPs increases also increases the chances of annihilation may be, some products around the material to absorb, but only because neutrinos weak interaction, penetration is very high, so you can fly to the earth's surface, and collide with the earth's surface material, and then produce charged particles. The charged particles can easily be captured by the detector. Neutrino detection is also sensitive to spin independent scattering cross sections. For ordinary WIMP particles, indirect detection is better than direct detection. In addition to neutrinos, there are many other particles may be a sign of dark matter annihilation. Compared with direct detection, a lot of abnormal signals have been reported in the indirect detection experiments, some of which are thought to be evidence of the existence of dark matter.

\section{Introduction to little Higgs T-parity model}

Compared to the little Higgs model, the little Higgs T-parity model is constructed based on the global symmetry of SU(5)/SO(5). The $\Sigma$ field of makes the SU(5)breaking into $S O(5)$. The Vacuum expectation value can be expressed by:

$$
\Sigma_{0}=\left(\begin{array}{lllll}
0 & 0 & 0 & 1 & 0 \\
0 & 0 & 0 & 0 & 1 \\
0 & 0 & 1 & 0 & 0 \\
1 & 0 & 0 & 0 & 0 \\
0 & 1 & 0 & 0 & 0
\end{array}\right)
$$

The subgroup $\left[S U(2)_{1} \times U(1)_{1}\right] \times\left[S U(2)_{2} \times U(1)_{2}\right]$ of $S U(5)$ is normal. The generator of the normal symmetry is:

Also, we have:

$$
Q_{1}^{a}=\left(\begin{array}{ccc}
\sigma^{a} / 2 & 0_{2} & 0_{2 \times 2} \\
0_{2}^{T} & 0 & 0_{2}^{T} \\
0_{2 \times 2} & 0_{2} & 0_{2 \times 2}
\end{array}\right) \text { and } Q_{2}^{a}=\left(\begin{array}{ccc}
0_{2 \times 2} & 0_{2} & 0_{2 \times 2} \\
0_{2}^{T} & 0 & 0_{2}^{T} \\
0_{2 \times 2} & 0_{2} & -\sigma^{a} / 2
\end{array}\right)
$$

$$
Y_{1}=\operatorname{diag}(3,3,-2,-2,-2) / 10, Y_{2}=\operatorname{diag}(2,2,2,-3,-3) / 10
$$

In the above formulations, $\sigma^{a}$ is Pauli matrix; $V E V$ makes $\left[S U(2)_{1} \times U(1)_{1}\right] \times$ $\left[S U(2)_{2} \times U(1)_{2}\right]$ break to its diagonal subgroup.

The transformation of the $\Sigma$ field by the function of $S U(5)$ :

$$
\Sigma \rightarrow V \Sigma V^{T}
$$

Then, we have:

$$
\mathrm{L}=\frac{f^{2}}{8} \operatorname{Tr}\left(D_{\mu} \Sigma\right)^{\dagger}\left(D_{\mu} \Sigma\right)
$$

In the above formulation, the covariant derivatives of $\left[S U(2)_{1} \times U(1)_{1}\right] \times$ $\left[S U(2)_{2} \times U(1)_{2}\right]$ is:

$$
D_{\mu} \Sigma=\partial_{\mu} \Sigma-\mathrm{i} \sum_{A=1,2}\left[\bar{g}_{A}\left(W_{A_{\mu}}^{a} Q_{A}^{a} \Sigma+\Sigma Q_{A}^{a} W_{A_{\mu}}^{a}\right)+\bar{g}_{A}^{\prime}\left(B_{A_{\mu}} Y_{A} \Sigma+\Sigma Y_{A} B_{A_{\mu}}\right)\right]
$$


$W_{A_{\mu}}^{a}$ and $B_{A_{\mu}}(A=1,2)$ are specification boson; $\bar{g}$ and $\bar{g}^{\prime}$ are normal coupling constant. The relationship between them is:

$$
1 / g^{2}=1 / \bar{g}_{1}^{2}+1 / \bar{g}_{2}^{2}, 1 / g^{\prime 2}=1 / \bar{g}_{1}^{\prime 2}+1 / \bar{g}_{2}^{\prime 2} .
$$

\section{Direct detection of dark matter based on little Higgs model}

Based on the detection of dark matter in the ground, it can be divided into indirect detection and direct detection. Direct detection is based on the DM and nucleon elastic scattering cross sections to determine the number of DM cases. The calculation model of the LHMT is obtained and a certain unit of nuclear reaction rate.

If the heavy photons are regarded as the only component of cold dark matter, the DM signal quantity will be determined by the intensity of the interaction between the photons and protons, the DM dispersion velocity and the density around the solar system. The rate of event generationunder the ground experiment is as follows:

$$
\Delta \mathrm{n}=\mathrm{N}<\sigma>n \bar{v} \Delta \mathrm{t} \varepsilon
$$

In the formulation, $\mathrm{N}$ is the number of protons in the detection instrument of the target; $\mathrm{n}=\rho_{0} / \mathrm{M}_{\mathrm{A}_{\mathrm{H}}}\left(\rho_{0}=0.3 \mathrm{GeV} / \mathrm{cm}^{3}\right)$ is the number density of the local area; $\langle\sigma v\rangle \cong\langle\sigma\rangle \overline{\mathrm{v}}$ is scattering cross section; $\overline{\mathrm{v}}=\sqrt{\left\langle\mathrm{v}^{2}\right\rangle}$ is the discretevelocity; $\Delta \mathrm{t}$ is the detection time; $\varepsilon$ is the detection efficiency. We select the $1000 \mathrm{~kg}$ water as the experiment sample.

In the experiment, the scattering cross section can be expressed as:

$$
<\sigma\left(v_{i}\right)>=\int_{0}^{\infty} f\left(v_{1}\right) \sigma\left(v_{1}\right) d^{3} v_{1}
$$

In the above formulation, $v_{1}=|v|$ is the speed in the laboratory coordinate system. $f\left(v_{1}\right) d^{3} v_{1}=4 \pi \exp \left(-v_{1}^{2} / v_{0}^{2}\right) /\left(\pi^{3 / 2} v_{0}^{2}\right) v_{1}^{2} d v_{1}$ is the Maxwell velocity distribution in the local area.

The differential scattering cross section can be expressed as:

$$
\left(\frac{d \sigma\left(v_{1}\right)}{d \cos \theta}\right)_{\mathrm{CM}}=\frac{\left|p_{4}\right|}{4 E_{A_{\mathrm{H}}} E_{\mathrm{P}}\left|v_{A_{\mathrm{H}}}-v_{\mathrm{P}}\right|} \frac{|M|^{2}}{2 \pi \cdot 4 \sqrt{S}}
$$

The quality of Higgs is $300 \mathrm{GeV}$, and the absolute value of dark matter can be obtained by the following formulation:

The Feynman diagram is drawn as follows:

$$
\left|p_{4}\right|=\left|p_{1}\right|=M_{\mathrm{P}} v_{1} /\left(M_{A_{\mathrm{H}}}+M_{\mathrm{P}}\right) \text {. }
$$




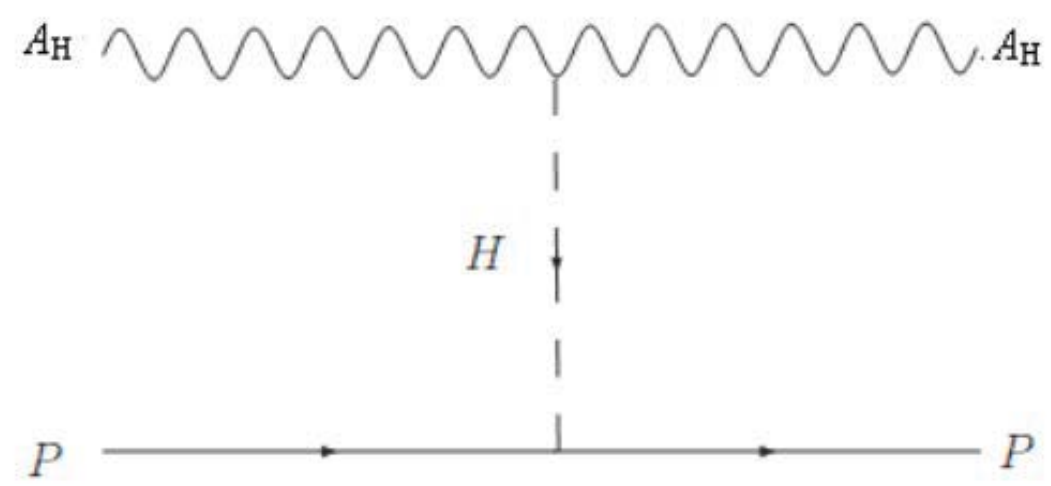

Fig. 1: The Feynman diagram of elastic collision between DM and proton

\section{Conclusion}

Generally speaking, the dark matter is difficult to be detected. This paper completes the task of direct detection of the dark matter by the Higgs T-parity model. The study of the dark matter needs the joint effort from different academic fields. We are looking forward some new methods to directly detect the dark matter.

\section{References}

[1]QiaoQingpeng, Zhang Xiangdan, Zhao Xianlin, EffectofInitialConditionsvsResidualDensityofLittlestHiggsModelDarkMatter, JournalofHenanInstituteofEducation(NaturalScienceEdition), 19(2), pp. 13-15, 2010.

[2]GaoXin, Kang Zhaofeng, Li Tianjun, Introduction to the dark matter models, ScientiaSinica(Physica,Mechanica\&Astronomica), 41(12), pp. 1396-1401, 2011. [3]Zhou Yufeng, Progresses in the study of the nature of dark matter anddetections, ScientiaSinica(Physica,Mechanica\&Astronomica), 45(4), pp. 1-21, 2015.

[4]GaoYinhao, Cao Jun, Production of charged scalars from the littlest Higgs model associated with top quark at LHC, Journal of Henan Institute of Science and Technology, 43(1), pp. 45-48, 2015. 